\title{
PRIOR FLARING AS A COMPLEMENT TO FREE MAGNETIC ENERGY FOR FORECASTING SOLAR ERUPTIONS
}

\author{
David A. Falconer ${ }^{1,2}$, Ronald L. Moore ${ }^{1}$, Abdulnasser F. Barghouty ${ }^{1}$, and Igor KhaZanov ${ }^{2}$ \\ ${ }^{1}$ ZP13 MSFC/NASA, Huntsville, AL 35812, USA \\ ${ }^{2}$ CSPAR, Cramer Hall/NSSTC, The University of Alabama in Huntsville, Huntsville, AL 35899, USA \\ Received 2012 March 27; accepted 2012 May 28; published 2012 September 4
}

\begin{abstract}
From a large database of (1) 40,000 SOHO/MDI line-of-sight magnetograms covering the passage of 1300 sunspot active regions across the $30^{\circ}$ radius central disk of the Sun, (2) a proxy of each active region's free magnetic energy measured from each of the active region's central-disk-passage magnetograms, and (3) each active region's fulldisk-passage history of production of major flares and fast coronal mass ejections (CMEs), we find new statistical evidence that (1) there are aspects of an active region's magnetic field other than the free energy that are strong determinants of the active region's productivity of major flares and fast CMEs in the coming few days; (2) an active region's recent productivity of major flares, in addition to reflecting the amount of free energy in the active region, also reflects these other determinants of coming productivity of major eruptions; and (3) consequently, the knowledge of whether an active region has recently had a major flare, used in combination with the active region's free-energy proxy measured from a magnetogram, can greatly alter the forecast chance that the active region will have a major eruption in the next few days after the time of the magnetogram. The active-region magnetic conditions that, in addition to the free energy, are reflected by recent major flaring are presumably the complexity and evolution of the field.
\end{abstract}

Key words: solar-terrestrial relations - Sun: coronal mass ejections (CMEs) - Sun: flares - Sun: surface magnetism

\section{INTRODUCTION}

The greatest disruptions of Earth's magnetosphere and ionosphere and the greatest solar energetic particle (SEP) storms in Earth's sector of the heliosphere are produced by the greatest explosive releases of magnetic energy on the face of the Sun (e.g., Suess \& Tsurutani 1998; Gopalswammy et al 2006). These releases are from the magnetic fields that have the greatest free energy, the extremely contorted fields in the chromosphere and low corona in certain large sunspot active regions (e.g., Canfield et al 1999). The contorted field explosively releases a fraction of its free energy by undergoing an eruption in which reconnection divides and unleashes the erupting field to simultaneously explode outward and implode inward (e.g., Moore et al. 2001). The outward exploding field becomes a large fast (super-Alfvénic) coronal mass ejection (CME), a huge plasmoid that explodes out into the solar wind, driving a bow shock that often produces an SEP storm that penetrates much of the heliosphere (e.g., Reames 1999; Gopalswammy et al. 2006). In this paper, a fast CME is one that has a frontal speed of at least $800 \mathrm{~km} \mathrm{~s}^{-1}$, the frontal speed of the slowest CMEs that produce appreciable SEP events (Kahler 2001). The release of magnetic energy in the imploding field produces a major flare. In this paper as in Schrijver (2007), a major flare is one that puts out a major burst of soft X-ray emission, a burst of GOES class $\mathrm{M}$ or $\mathrm{X}$.

Figure 1 shows a large fast CME blasting out into the solar wind. It was produced together with an X-class flare and had a speed of about $1100 \mathrm{~km} \mathrm{~s}^{-1}$ (Yashiro et al. 2004). Because the source active region was about $20^{\circ}$ east of central meridian, this CME was centered on lines of the solar wind's Parkerspiral magnetic field that passed far eastward of Earth at $1 \mathrm{AU}$. Thus, Earth-Moon space was magnetically shielded from most particles accelerated by the CME's bow shock. Even so, this CME produced a moderate SEP storm in Earth-Moon space (Yashiro et al. 2004). In addition, the flare's X-ray and EUV emission strongly disturbed the ionosphere. The ionosphere is similarly disturbed by any $\mathrm{M}$ or $\mathrm{X}$ flare that happens anywhere on the disk, regardless of whether the eruption also produces a CME (e.g., Suess \& Tsurutani 1998). Most M or X flares are produced together with a CME, and about half of these CMEs are fast CMEs (Falconer et al. 2011). The closer the source active region is to the disk center the more nearly Earth-centered the swath of the CME is likely to be, and the greater the CME's impact on the magnetosphere is likely to be (e.g., Moore et al. 2007).

Because of the disruptive and destructive effects that major flares and fast CMEs can have on global radio transmission, and on communication satellites and power grids caught unawares, advanced warning of these solar events is needed to prepare for disruptions and to safeguard these vital systems. This requires reliable forecasting of the chance that a solar active region will produce a fast CME and/or major flare in the coming day or so, forecasting based on observable indicators of the magnetic field's readiness to explode.

Previous work has established that proxies of an active region's free magnetic energy can be measured from the lineof-sight component of the magnetic field in the photosphere, provided the active region is within $\sim 30^{\circ}$ of disk center, close enough to disk center that the line-of-sight component adequately approximates the field's vertical component (Falconer et al 2003; Schrijver 2007). These free-energy proxies are measures of photospheric signatures of the free energy that is in the field above the photosphere. The proxy measures do not have units of energy, but each increases with the active region's free energy. We have found that the particular free-energy proxy used in this paper has a success rate of about $80 \%$ as a predictor of whether an active region will produce a CME in the coming few days (Falconer et al. 2008). The number of major flares and the number of fast CMEs produced by an active region in a day are each strongly correlated with the value of the active region's 


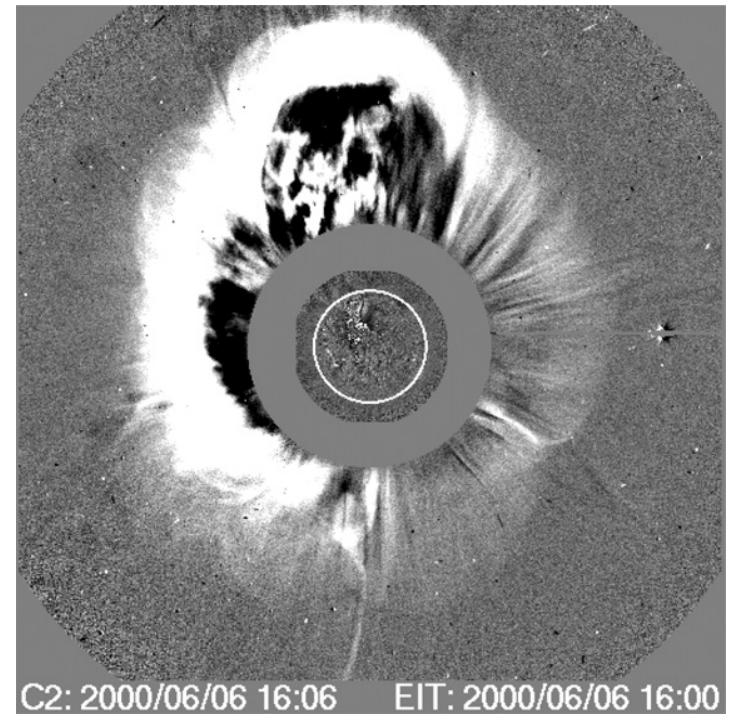

Figure 1. Large fast CME of 2000 June 6. This CME was produced in tandem with a GOES X-class flare by the explosion of magnetic field rooted in and around a large $\delta$ sunspot (in NOAA Active Region 9066) located at N20, $\mathrm{E} 18^{\circ}$ on the central face of the Sun. Shown here is a running-difference image from the $\mathrm{SOHO} / \mathrm{LASCO} / \mathrm{C} 2$ coronagraph.

free-energy proxy measured at the start of the day. This correlation for each of these two kinds of events forecasts from an active region's measured free-energy proxy the chance that the active region will produce an event of that kind in the coming day or so (Falconer et al. 2011).

It is observed that active regions that have had a major flare in the past day or so have more major flares in the coming day or so than active regions that have not recently had a major flare (Zirin 1988; Zirin \& Marquette 1991; Wheatland 2004; Welsch et al. 2009). It is also observed that the more flareproductive active regions tend to (1) have a complex multipolar arrangement of opposite-polarity magnetic flux rather than a single-bipole arrangement, and/or (2) be rapidly evolving via convective flux transport and/or flux emergence and/or flux cancellation (Svestka 1976; Zirin 1988; Zirin \& Marquette 1991; Welsch et al. 2009). Evidently, in addition to depending strongly on the active region's free energy, an active region's flare productivity also depends significantly on the complexity of the active region's field configuration, on the rate of evolution of the configuration, and on the occurrence of emergence and cancellation of flux in the active region. We therefore expect that an active region's recent production of major flares reflects these additional factors along with reflecting the free energy in the active region's magnetic field.

In this paper, we show that for active regions having prior major flaring and mid-range free energy, an active region's comingday major-flare-production chance and fast-CME-production chance forecast from the active region's free-energy proxy are much greater when the prior major flaring is taken into account instead of being ignored. This confirms that, in addition to depending strongly on the free energy, an active region's production of major flares and fast CMEs depends significantly on one or more other aspects of the active region's evolving magnetic field.

\section{DATA}

The data used in this study are from the large database that was compiled for the forecasting tool presented in Falconer et al.
(2011). This tool forecasts from an active region's free-energy proxy measured from a line-of-sight magnetogram the chance that the active region will have a major eruption during the next day or so after the time of the magnetogram. The database is from about 1300 sunspot active regions that occurred during 1996-2004, the first eight years of sunspot cycle 23. The database includes each active region's full-disk-passage history of production of major flares observed by GOES and SOHO/EIT and fast CMEs observed by $\mathrm{SOHO} / \mathrm{LASCO}$. Each active region's passage across the $30^{\circ}$ radius $\left(0.5 R_{\text {Sun }}\right)$ central disk was tracked in the full-disk line-of-sight magnetograms from SOHO/MDI, which have a cadence of 15 day $^{-1}$ (96 minutes), yielding a total of about 40,000 active-region magnetograms for the 1300 active regions. Along with the event-production history of each active region, the database has the value of the free-energy proxy measured from each of the active region's central-disk-passage magnetograms.

What we call the "gradient-weighted length of strong-field neutral line" is the free-energy proxy that we measured from each active-region magnetogram. This free-energy proxy is denoted by ${ }^{\mathrm{L}} \mathrm{WL}_{\mathrm{SG}}$ and is defined by ${ }^{\mathrm{L}} \mathrm{WL}_{\mathrm{SG}}=\int\left|\nabla B_{\mathrm{LOS}}\right| d l$, where the integral is the line integral on all "strong-field" intervals of the magnetogram's neutral lines, the intervals on which the transverse component of the potential field computed from the magnetogram is stronger than $150 \mathrm{G}$, and $\left|\nabla B_{\mathrm{LOS}}\right|$ is the absolute value of the line-of-sight field's gradient measured from the magnetogram. A magnetogram's neutral lines are the lines dividing opposite-polarity domains of the line-of-sight magnetic flux. On those lines the line-of-sight field is zero, changing from positive polarity (pointing toward the observer) to negative polarity (pointing away from the observer) with position across the line. The measurement uncertainty in the value of ${ }^{\mathrm{L}} \mathrm{WL}_{\mathrm{SG}}$ is determined from the uncertainties in the measured magnetogram as described in Falconer et al (2008).

The quantity ${ }^{\mathrm{L}} \mathrm{WL}_{\mathrm{SG}}$ is a proxy measure of an active region's free magnetic energy by virtue of (1) the observed strong correlation between the gradient of the line-of-sight field and the shear in the transverse field at neutral lines in vector magnetograms of active regions on the central disk (Falconer et al. 2003), and (2) the observed strong correlation between the amount of magnetic shear along the neutral line and the net electric current arching over the neutral line in bipolar active regions (Falconer et al. 2006). Because the net electric current in a bipolar active region directly corresponds to the free energy, it is certainly a proxy of the active region's free energy. Hence, because in both bipolar and multipolar active regions ${ }^{\mathrm{L}} \mathrm{WL}_{\mathrm{SG}}$ is strongly correlated with the amount of magnetic shear along the strong-field neutral lines, and in bipolar active regions the magnetic shear along the neutral line is strongly correlated with the net electric current, we infer that ${ }^{\mathrm{L}} \mathrm{WL}_{\mathrm{SG}}$ is a proxy measure of free magnetic energy for all sunspot active regions, bipolar and multipolar (Falconer et al. 2008).

One of the active-region magnetograms from which ${ }^{\mathrm{L}} \mathrm{WL}_{\mathrm{SG}}$ was measured for the database is shown in Figure 2. This is one of the measured magnetograms of the active region (AR 9066) from which the fast CME in Figure 1 exploded. It shows the active region's magnetic field about $15 \mathrm{hr}$ before the start of that explosion. It is from a full-disk MDI magnetogram and covers all of the active region's line-of-sight flux stronger than $100 \mathrm{G}$. The colored paths trace the strong-field intervals of the neutral lines. The strength of the gradient $\left(\left|\nabla B_{\mathrm{LOS}}\right|\right)$ at each point of the neutral line in these intervals is shown by the shade of color, the lighter the shade the stronger the gradient. In this way, Figure 2 


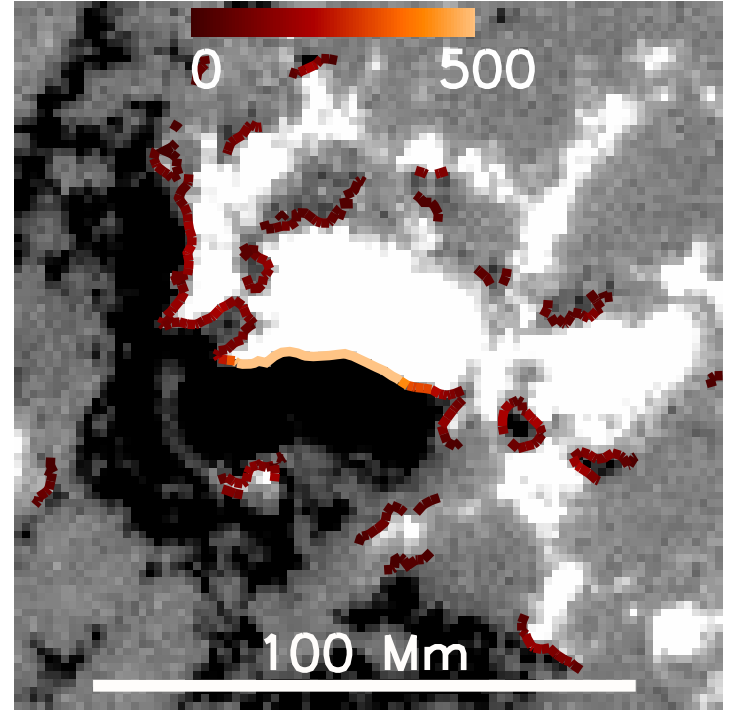

Figure 2. Strong-field intervals of the neutral lines in an MDI magnetogram of the $\delta$-sunspot active region from which the CME in Figure 1 exploded. The polarity, strength, and distribution of the line-of-sight flux are mapped by the gray-scale image. The polarity is positive (negative) in light (dark) areas. The strong-field intervals of the neutral lines are traced by the colored curves. The color gives the strength of the gradient of the line-of-sight field $\left(\left|\nabla B_{\mathrm{LOS}}\right|\right)$ at these neutral lines in units of $\mathrm{G} \mathrm{Mm}^{-1}$. The lightest color is for $\left|\nabla B_{\mathrm{LOS}}\right| \geqslant$ $500 \mathrm{G} \mathrm{Mm}^{-1}$, the range of the extreme gradients at the neutral line through the large $\delta$ sunspot.

shows that by far the strongest field gradients across neutral lines in this active region were in the $\delta$ sunspot in the center of the active region, and hence that most of the value of ${ }^{\mathrm{L}} \mathrm{WL}_{\mathrm{SG}}$ for this active region came from this big $\delta$ sunspot.

\section{FORECASTING FROM FREE-ENERGY PROXY ALONE}

In this section, we present our method of obtaining, from the database for a given type of event, a forecasting curve which gives for an active region the event rate expected during the next day or so as a function of the active region's present measured free-energy proxy ${ }^{\mathrm{L}} \mathrm{WL}_{\mathrm{SG}}$. This is the method used in Falconer et al. (2011). Here, we employ the method to obtain two forecasting curves: one for major-flare productivity and the other for fast-CME productivity.

Each forecasting curve is obtained from a histogram of the observed event rate as a function of ${ }^{\mathrm{L}} \mathrm{WL}_{\mathrm{SG}}$. In this work, for forecasting from ${ }^{\mathrm{L}} \mathrm{WL}_{\mathrm{SG}}$ alone, the histogram we use has 20 equally populated bins, each bin spanning about 2000 consecutive ordered values of ${ }^{\mathrm{L}} \mathrm{WL}_{\mathrm{SG}}$, each value measured from a separate active-region magnetogram. From the database, we have for each measured magnetogram the number of major flares and the number of fast CMEs produced by the active region in the next $24 \mathrm{hr}$ after the time of the magnetogram. For major flares, for example, the number of the next $24 \mathrm{hr}$ major flares summed over the magnetograms in a bin and divided by the number of magnetograms in the bin is the observed average next $24 \mathrm{hr}$ rate of production of major flares by active regions having measured values of ${ }^{\mathrm{L}} \mathrm{WL}_{\mathrm{SG}}$ in the span of that bin. From the 20-bin histogram, we obtain a 20-point log-log plot of the observed average next $24 \mathrm{hr}$ event rate versus the average value of ${ }^{\mathrm{L}} \mathrm{WL}_{\mathrm{SG}}$ in each bin. In Figure 3, the first plot is the one obtained for coming major-flare productivity, and the second one is for coming fast-CME productivity.

In Figure 3, the diamonds show the coming-day event rate for the bins in which the average ${ }^{\mathrm{L}} \mathrm{WL}_{\mathrm{SG}}$ is $10^{4} \mathrm{G}$ or greater, and the asterisks show the rate for the bins of smaller ${ }^{\mathrm{L}} \mathrm{WL}_{\mathrm{SG}}$. The error bar of each point shows the $1 \sigma$ uncertainty in the coming-day event rate found for that bin. We estimated this uncertainty by using Poisson statistics (Iman \& Conover 1983) and conservatively assuming that for each active region only magnetograms at least a day apart (about 1 in 15 of the active region's magnetorams from MDI) give non-redundant samples of the active region's coming-day event rate (Falconer et al. 2011). The Poison statistics take into account both the number of non-redundant magnetograms in the bin and the fraction of these for which the magnetogram's active region had an event in the coming $24 \mathrm{hr}$. Because the fraction of active regions that produce major flares and/or fast CMEs decreases with decreasing ${ }^{\mathrm{L}} \mathrm{WL}_{\mathrm{SG}}$ and approaches zero as ${ }^{\mathrm{L}} \mathrm{WL}_{\mathrm{SG}}$ decreases below $10^{4} \mathrm{G}$, the error bars become excessively large as ${ }^{\mathrm{L}} \mathrm{WL}_{\mathrm{SG}}$ decreases below $10^{4} \mathrm{G}$ : the uncertainty becomes increasingly larger than twice the measured value of the coming-day event rate. For this reason, we obtain our forecasting curves by fitting only the points for ${ }^{\mathrm{L}} \mathrm{WL}_{\mathrm{SG}}$ above $10^{4} \mathrm{G}$, the diamond points.

In each of the two plots in Figure 3, the straight line is a leastsquares linear fit to the diamond points in the log-log space of the plot. That is, the fit is for the linear dependence of $\log R$ on $\log { }^{\mathrm{L}} \mathrm{WL}_{\mathrm{SG}}$, where $R$ is the event rate in events per day. Both the fit and the uncertainty in the fit come from both the $\log R$ values of the points and their uncertainties: the greater the uncertainty in $\log R$ the smaller the weight of the point in the fit. In each plot, the fit's slope and the slope's $1 \sigma$ uncertainty are given in the upper left corner. These fits show that for both coming major-flare productivity and coming fast-CME productivity, the increase in the coming event rate $R$ with increasing freeenergy proxy ${ }^{\mathrm{L}} \mathrm{WL}_{\mathrm{SG}}$ is approximately a constant-index power law: on the least-squares line, $(\log R) /\left(\log { }^{\mathrm{L}} \mathrm{WL}_{\mathrm{SG}}\right)=\alpha$, or $R \propto\left({ }^{\mathrm{L}} \mathrm{WL}_{\mathrm{SG}}\right)^{\alpha}$, where the power-law index $\alpha$ is the slope of the line.

The two fit lines in Figure 3 are our forecasting curves for forecasting an active region's coming major flare productivity and coming fast-CME productivity. For a presently observed active region, from the active region's value of ${ }^{\mathrm{L}} \mathrm{WL}_{\mathrm{SG}}$ (measured from a line-of-sight magnetogram (such as from the Solar Dynamics Observatory $(S D O)$ ) and calibrated to give the value that would be measured from an MDI magnetogram), the forecasting curves give the active region's expected rate of production of major flares and expected rate of production of fast CMEs in the coming few days. For any chosen time interval $t$ spanning no more than a few days from the time of the measured magnetogram, the forecast percent chance $P$ that the active region will produce a major flare (fast $\mathrm{CME}$ ) during that interval is given by $P=\left(1-e^{-R t}\right) 100 \%$, where $R$ is the coming event rate forecast from the active region's value of ${ }^{\mathrm{L}} \mathrm{WL}_{\mathrm{SG}}$ via the forecasting curve for major-flare (fast-CME) production (Wheatland 2001; Moon et al. 2001).

\section{CORRELATION OF PRIOR FLARING WITH FREE MAGNETIC ENERGY}

As was mentioned in Section 1, observers have long noticed that active regions that have had a major eruption (major flare and/or fast CME) in the past day or so are much more likely to have a major eruption in the next day or so than those that have had no recent major eruption (e.g., Zirin \& Marquette 1991). In this section, we show that an active region's rate of production of major flares in the past $24 \mathrm{hr}$ and its rate of production of major flares in the coming $24 \mathrm{hr}$ are about equally strongly correlated 

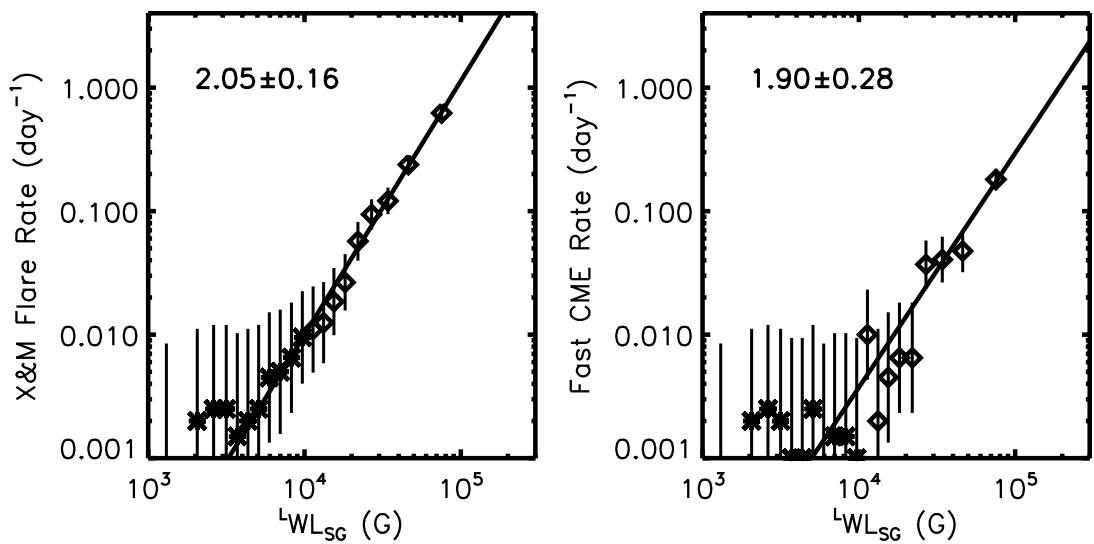

Figure 3. Empirical forecasting curves for forecasting for the next day or so an active region's rate of production of major flares (left plot) and fast CMEs (right plot) from the active region's free-energy proxy ${ }^{\mathrm{L}} \mathrm{W} \mathrm{L}_{\mathrm{SG}}$ measured from a magnetogram. See the text.
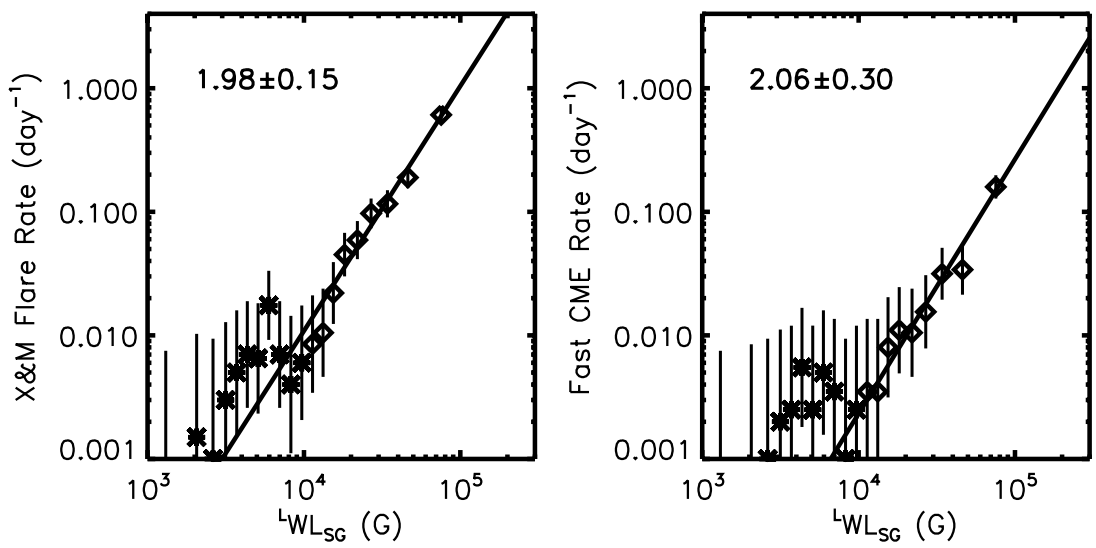

Figure 4. Correlation of prior rate of major-flare production (left plot) and prior rate of fast-CME production (right plot) with the active region's present value of the free-energy proxy ${ }^{\mathrm{L}} \mathrm{WL}_{\mathrm{SG}}$. The format is the same as in Figure 3. See the text.

with the active region's present value of the free-energy proxy ${ }^{\mathrm{L}} \mathrm{WL}_{\mathrm{SG}}$, and likewise for fast-CME productivity. This brings into question whether the observed strong correlation of an active region's coming major-eruption productivity with its recent major-eruption productivity significantly reflects any dependence of coming major-eruption productivity on activeregion conditions other than the free magnetic energy. Our answer to this question is the point of this paper and is presented in Section 5.

The two plots in Figure 4 directly correspond to the two plots in Figure 3. The only difference is that Figure 4 shows the correlations between the free-energy proxy ${ }^{\mathrm{L}} \mathrm{WL}_{\mathrm{SG}}$ and the observed event rate for the past $24 \mathrm{hr}$ for major flares (left plot) and fast CMEs (right plot), whereas the corresponding plots in Figure 3 show these correlations for the coming $24 \mathrm{hr}$. For major flares, the slope of the power-law fit is $1.98 \pm 0.15$ for prior flaring (Figure 4) and $2.05 \pm 0.16$ for coming flaring (Figure 3). For fast CMEs, the slope is $2.06 \pm 0.30$ for prior productivity (Figure 4) and $1.90 \pm$ 0.28 for coming productivity (Figure 3 ). Thus, within their $1 \sigma$ uncertainties, the slope of the fits is the same for all four plots, and is about 2 . This result shows that an active region's prior productivity and coming productivity of major eruptions both depend strongly on the active region's present free magnetic energy, and that the dependence is about equally strong for both. This suggests that an active region's prior major flaring might not be significantly separate from the freeenergy proxy ${ }^{\mathrm{L}} \mathrm{WL}_{\mathrm{SG}}$ as an indicator of coming major-eruption productivity.

\section{FORECASTING FROM FREE-ENERGY PROXY AND PRIOR FLARING}

In this section, we show that, in addition to being an indicator of the free energy in an active region's magnetic field, an active region's recent major-flare productivity is an indicator of other conditions that are significant determinants of the active region's coming productivity of major eruptions. To show this, we divide our set of 40,000 active-region magnetograms into two subsets: the magnetograms of active regions that had a major flare within $24 \mathrm{hr}$ before the time of the magnetogram, and the magnetograms of active regions that did not have a major flare in the past $24 \mathrm{hr}$. We refer to these two subsets as the "priorflaring subset" and the "no-prior-flaring" subset. By the same method as we obtained the plots in Figure 3 from the full set of magnetograms, from each subset we obtain the corresponding plots of coming-day rate of production of major flares and coming-day rate of production of fast CMEs as functions of the free-energy proxy ${ }^{\mathrm{L}} \mathrm{WL}_{\mathrm{SG}}$. These plots are shown in Figure 5.

In each plot in Figure 5, the points, their straight-line fit, and its slope are shown in red for the prior-flaring subset and in blue for the no-prior-flaring subset. As in Figure 3, the points having ${ }^{\mathrm{L}} \mathrm{WL}_{\mathrm{SG}} \geqslant 10^{4} \mathrm{G}$ are diamonds, the points having ${ }^{\mathrm{L}} \mathrm{WL}_{\mathrm{SG}}<$ $10^{4} \mathrm{G}$ are asterisks, and the power-law line is fit to the diamond points. The black line in each plot in Figure 5 is the powerlaw line from the corresponding plot of Figure 3 for the full set. Because only a small fraction of all active regions produce major flares, only about $4 \%$ of the full set of 40,000 magnetograms is in the prior-flaring subset and about $96 \%$ are in the no-prior- 

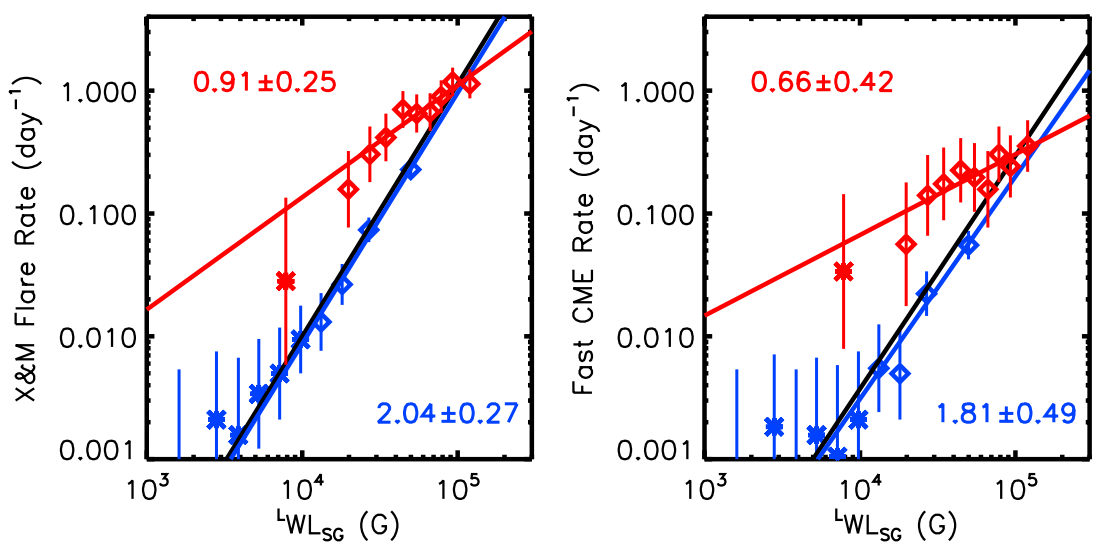

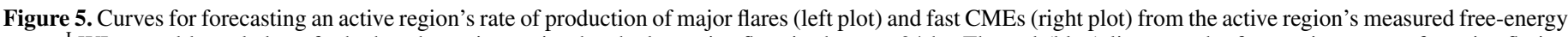

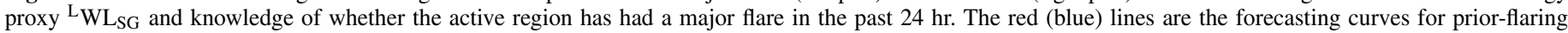
(no-prior-flaring) active regions. See the text.

flaring subset. Thus, for the plots for the no-prior-flaring subset we divided this subset into 20 equally populated bins as in Figure 3 for the full set. But, because the prior-flaring subset is 25 times smaller than the full set, to keep the error bars for the prior-flaring subset less than about twice the event rate for the points having ${ }^{\mathrm{L}} \mathrm{WL}_{\mathrm{SG}} \geqslant 10^{4} \mathrm{G}$, we divided the prior-flaring subset into 10 equally populated bins instead of 20 .

Not surprisingly, the Figure 5 plots for the no-prior-flaring subset show for both major-flare and fast-CME coming-day productivity the increase in coming-day event rate with increasing free-energy proxy ${ }^{\mathrm{L}} \mathrm{WL}_{\mathrm{SG}}$ follows a power law that is nearly the same as for the full set. In contrast, for each of the two kinds of events, the plot for the prior-flaring subset follows a power-law trend that is clearly different from the power law for no-priorflaring active regions. Over most of the range of ${ }^{\mathrm{L}} \mathrm{WL}_{\mathrm{SG}}$, the coming-day event rates for the points from the prior-flaring subset are significantly higher than for points of the same ${ }^{\mathrm{L}} \mathrm{WL}_{\mathrm{SG}}$ value from the no-prior-flaring subset. Also, the slope of the power-law fit for the prior-flaring subset's significantly flatter than that for the no-prior-flaring subset. Hence, the forecasting curve for prior-flaring active regions (the red line) intersects the forecasting curve for no-prior-flaring active regions (the blue line) near the high end of the ${ }^{\mathrm{L}} \mathrm{WL}_{\mathrm{SG}}$ range, at about $10^{5} \mathrm{G}$, and lies increasingly farther above the no-prior-flaring forecasting curve with decreasing ${ }^{\mathrm{L}} \mathrm{WL}_{\mathrm{SG}}$ below $10^{5} \mathrm{G}$.

Thus, for both major-flare and fast-CME coming productivity, Figure 5 shows that active regions having values of the freeenergy proxy ${ }^{\mathrm{L}} \mathrm{WL}_{\mathrm{SG}}$ in the range from $\sim 10^{4} \mathrm{G}$ to $\sim 5 \times 10^{4} \mathrm{G}$ have a much greater chance of producing an event in the coming day or so if they have produced a major flare in the past day than if they have not. This means that an active region's prior major flaring is an indicator of conditions in an active region other than the free magnetic energy that are significant determinants of the active region's coming productivity of major flares and fast CMEs.

\section{DISSCUSION}

Figure 5 shows that an active region having free energy less than the amount corresponding to a value of $\sim 10^{4} \mathrm{G}$ for its freeenergy proxy ${ }^{\mathrm{L}} \mathrm{WL}_{\mathrm{SG}}$ has no more than a few percent chance of producing a major flare and/or fast CME in the coming day. This reflects the statistic that hardly any active regions having ${ }^{\mathrm{L}} \mathrm{WL}_{\mathrm{SG}}$ $<10^{4} \mathrm{G}$ produce a major flare and/or fast CME: of the hundreds of active regions in our database that had ${ }^{\mathrm{L}} \mathrm{WL}_{\mathrm{SG}}<10^{4} \mathrm{G}$, less than $1 \%$ produced a major flare and/or fast CME. We interpret this to mean that active regions having ${ }^{\mathrm{L}} \mathrm{WL}_{\mathrm{SG}} \sim 10^{4} \mathrm{G}$ are hardly able to have a major eruption simply because they have hardly enough free energy, and that active regions having ${ }^{\mathrm{L}} \mathrm{WL}_{\mathrm{SG}}$ much less than $10^{4} \mathrm{G}$ do not have enough free energy to have a major eruption.

Figure 5 also shows that at ${ }^{\mathrm{L}} \mathrm{WL}_{\mathrm{SG}} \sim 10^{4} \mathrm{G}$, an active region from the small minority that have had a major flare in the past day are 5-10 times more likely to have a major eruption in the coming day than an active region from the large majority that have not had a major flare in the past day. As ${ }^{\mathrm{L}} \mathrm{WL}_{\mathrm{SG}}$ increases above $10^{4} \mathrm{G}$ the difference between the two forecasting curves decreases until the two lines intersect just beyond ${ }^{\mathrm{L}} \mathrm{WL}_{\mathrm{SG}}=$ $10^{5} \mathrm{G}$. As ${ }^{\mathrm{L}} \mathrm{WL}_{\mathrm{SG}}$ increases beyond its value at the intersection (at about $1.5 \times 10^{5} \mathrm{G}$ ), the steeper expected-event-rate line for no-prior-flaring active regions rises above the flatter expectedevent-rate line for prior-flaring active regions. In our database, for active regions having ${ }^{\mathrm{L}} \mathrm{WL}_{\mathrm{SG}}$ above a given lower bound, the fraction of active regions that are prior-flaring active regions steadily increases from about $4 \%$ when the lower bound is near the bottom of the range of ${ }^{\mathrm{L}} \mathrm{WL}_{\mathrm{SG}}\left(\right.$ at $\left.\sim 10^{3} \mathrm{G}\right)$ to about $96 \%$ when the lower bound is at the intersection of the two forecasting curves. We interpret these results as follows. Active regions having ${ }^{\mathrm{L}} \mathrm{WL}_{\mathrm{SG}} \sim 10^{4} \mathrm{G}$ or greater have enough free energy for a major eruption and become increasingly more easily destabilized to have a major eruption with increasing ${ }^{\mathrm{L}} \mathrm{WL}_{\mathrm{SG}}$ and corresponding increasing free energy. Active regions having ${ }^{\mathrm{L}} \mathrm{WL}_{\mathrm{SG}} \sim 10^{5} \mathrm{G}$ have so much free energy that they can hardly keep from exploding. That is, for an active region having that much free energy it is unlikely that a major eruption will not be triggered in the span of a day. Consequently, the rare active region that has ${ }^{\mathrm{L}} \mathrm{WL}_{\mathrm{SG}}>1.5 \times 10^{5} \mathrm{G}$ and has not had a major eruption in the past day is somewhat more likely to have a major eruption in the coming day than an active region of the same large ${ }^{\mathrm{L}} \mathrm{WL}_{\mathrm{SG}}$ that has had a major eruption in the past day, simply because an active region's chance of having another major eruption increases with increasing time since its last major eruption.

In each of the two plots in Figure 5, while the prior-flaring red line is flatter than the no-prior-flaring blue line, its slope is more than $1 \sigma$ steeper than entirely flat (horizontal). If we ignored ${ }^{\mathrm{L}} \mathrm{WL}_{\mathrm{SG}}$ and used only the knowledge that an active region has produced a major flare in the past $24 \mathrm{hr}$ to forecast the active region's coming rates of production of major flares and fast CMEs, the forecast rate for each kind of event would be the average coming rate found for all of the prior-flaring active 
regions in our sample. For the prior-flaring active regions in our sample, the average next-day rate of production of major flares is $0.61 \pm 0.07$ major flares per day and the average next-day rate of production of fast CMEs is $0.19 \pm 0.04$ fast CMEs per day. These rates are within a factor of two of the observed rates in Figure 5 for very nonpotential prior-flaring active regions, those having ${ }^{\mathrm{L}} \mathrm{WL}_{\mathrm{SG}} \sim 10^{5} \mathrm{G}$. Thus, for those very nonpotential prior-flaring active regions, the coming rates of major flares and fast CMEs could be forecast fairly well from knowledge of prior flaring alone, and ignoring ${ }^{\mathrm{L}} \mathrm{WL}_{\mathrm{SG}}$. But for prior-flaring active regions that are only weakly to moderately nonpotential, those having ${ }^{\mathrm{L}} \mathrm{WL}_{\mathrm{SG}} \sim 10^{4} \mathrm{G}$, Figure 5 shows that the above average coming rate for prior-flaring active regions exceeds the observed rate (the red line) by a factor of $\sim 5$ for major flares and a factor of $\sim 3$ for fast CMEs. For active regions having ${ }^{\mathrm{L}} \mathrm{WL}_{\mathrm{SG}} \sim 10^{4} \mathrm{G}$, the average rates for prior-flaring active regions are closer to the observed rates for prior-flaring active regions than are the rates forecast from ${ }^{\mathrm{L}} \mathrm{WL}_{\mathrm{SG}}$ alone (the black lines in Figure 5). Even so, as Figure 5 clearly shows, using the combination of both ${ }^{\mathrm{L}} \mathrm{WL}_{\mathrm{SG}}$ and knowledge that the active region produced a major flare in the past day yields a significantly more accurate forecast (red lines) than forecasting either from ${ }^{\mathrm{L}} \mathrm{WL}_{\mathrm{SG}}$ alone or from knowledge of prior flaring alone.

We expect that the difference in coming major-eruption rate signaled by whether an active region has had recent major flaring reflects the combined ability of the complexity and evolution of the active region's magnetic field to induce the field to erupt. Whether the complexity of the field configuration or the rate of evolution of the field is the more important aspect for unleashing major eruptions remains obscure and needs further observational study.
This paper resulted from research funded by NASA's Heliophysics Division, NSF's Division of Atmospheric Sciences, and AFOSR's Multi-University Research Initiative. The paper was improved by helpful comments from the referee.

\section{REFERENCES}

Canfield, R. C., Hudson, H. S., \& McKenzie, D. E. 1999, Geophys. Res. Lett., 26, 627

Falconer, D., Barghouty, A. F., Khazanov, I., \& Moore, R. 2011, Space Weather, 9, S04003

Falconer, D. A., Moore, R. L., \& Gary, G. A. 2003, J. Geophys. Res., 108, 1380

Falconer, D. A., Moore, R. L., \& Gary, G. A. 2006, ApJ, 644, 1258

Falconer, D. A., Moore, R. L., \& Gary, G. A. 2008, ApJ, 689, 1433

Gopalswammy, N., Mewaldt, R., \& Torsti, J. 2006, Solar Eruptions and Energetic Particles (Washington, DC: AGU)

Iman, R. L., \& Conover, W. J. 1983, A Modern Approach to Statistics (New York: Wiley)

Kahler, S. W. 2001, J. Geophys. Res., 106, 20947

Moon, Y.-J., Choe, G. S., Yun, H. I., \& Park, Y. D. 2001, J. Geophys. Res., 106, 29951

Moore, R. L., Sterling, A. C., Hudson, H. S., \& Lemen, J. R. 2001, ApJ, 552, 833

Moore, R. L., Sterling, A. C., \& Suess, S. T. 2007, ApJ, 668, 1221

Reames, D. V. 1999, Space Sci. Rev., 90, 412

Schrijver, C. J. 2007, ApJ, 655, L117

Suess, S. T., \& Tsurutani, B. T. (ed.) 1998, From the Sun: Auroras, Magnetic Storms, Solar Flares, Cosmic Rays (Washington, DC: AGU)

Svestka, Z. 1976, Solar Flares (Berlin: Springer)

Welsch, B. T., Li, Y., Schuck, P. W., \& Fisher, G. H. 2009, ApJ, 705, 821

Wheatland, M. S. 2001, Sol. Phys., 203, 87

Wheatland, M. S. 2004, ApJ, 609, 1134

Yashiro, S., Gopalswamy, N., Michalek, G., et al. 2004, J. Geophys. Res., 109, A07105

Zirin, H. 1988, Astrophysics of the Sun (Cambridge: Cambridge Univ. Press)

Zirin, H., \& Marquette, W. 1991, Sol. Phys., 131, 149 